\title{
Etude de la régénération naturelle contrôlée en forêt tropicale humide de Guadeloupe I. - Revue bibliographique, milieu naturel
et élaboration d'un protocole expérimental
}

\author{
M. DUCREY (*) et P. LABBE \\ I.N.R.A., Station de Recherches forestières \\ Centre de Recherches agronomiques des Antilles et de la Guyane \\ Domaine Duclos, F 97170 Petit-Bourg, Guadeloupe
}

\begin{abstract}
Résumé
Après une revue bibliographique des études sur la régénération naturelle des forêts tropicales, les auteurs donnent des informations sur la forêt hygrophile de Guadeloupe.

Cette forêt présente l'originalité dans le monde tropical d'être riche en un nombre limité d'espèces précieuses.

Deux espèces principales : le gommier (Dacryodes excelsa) et le bois rouge carapate (Amanoa caribaea) représentent respectivement 20,7 p. 100 et 12,6 p. 100 du nombre de tiges total.

Six autres espèces, bien que moins fréquentes, sont intéressantes : le marbri (Richeria grandis), l'acajou blanc (Simaruba amara), le palétuvier jaune (Symphonia globulifera), le mauricif (Byrsonima coriacea), le résolu (Chimarrhis cymosa) et le bois doux chypre (Phoebe elongata).
\end{abstract}

L'ensemble de ces 8 espèces représente 48 p. 100 du nombre de tiges total et 64 p. 100 de la surface terrière totale.

Cette particularité rend possible l'idée d'utiliser une technique proche de la régénération naturelle par coupes progressives et apparentée au «Tropical shelterwood system» pour le renouvellement et l'enrichissement de cette forêt en essences précieuses.

L'expérimentation mise en place entre 1979 et 1981 et fondée sur une description précise du milieu forestier, comporte 5 traitements :

- 1 parcelle ( 2 ha) avec une coupe densemencement forte, 1 coupe secondaire à 3 ans et 1 coupe définitive à 6 ans ;

- 1 parcelle ( 2 ha) avec une coupe d'ensemencement forte, 1 coupe secondaire à 4 ans et 1 coupe définitive à 8 ans;

- 1 parcelle ( $2 \mathrm{ha})$ avec une coupe d'ensemencement faible, 2 coupes secondaires à 3 et 6 ans et 1 coupe définitive à 9 ans ;

(\%) Adresse actuelle : I.N.R.A., Station de Sylviculture méditerranéenne, avenue A. Vivaldi, 84000 Avignon. 
- 1 parcelle ( 2 ha) avec une coupe d'ensemencement faible, 2 coupes secondaires à 4 et 8 ans et 1 coupe définitive à 12 ans;

- 1 parcelle témoin ( 1 ha) sous forêt naturelle.

Elle a été répétée en 4 endroits différents de la forêt hygrophile.

Le but principal est d'étudier la régénération naturelle, la survie et la croissance des semis des 8 espèces principales. On utilisera aussi les différences écologiques entre parcelles, créées par les traitements sylvicoles, pour préciser le tempérament de ces espèces.

\section{Introduction}

L'exploitation d'un massif forestier pour la production de bois pose toujours le problème de la reconstitution de la forêt.

$\mathrm{Si}$, dans les pays tempérés, les techniques de régénération sont relativement bien connues, il n'en est pas de même et de loin dans le monde tropical.

En effet, on assiste beaucoup plus à une exploitation minière qu'à une sylviculture raisonnée et à une gestion rigoureuse des forêts tropicales. C'est pourquoi, dans ce milieu qui représente environ 60 p. 100 de la couverture forestière mondiale, soit 2,5 milliards d'hectares, et qui pourrait de ce fait paraître inépuisable, de graves problèmes comme la surexploitation ou la déforestation abusive se font sentir chaque jour.

La destruction des forêts denses tropicales est actueïzment évaluée à 5,5 millions d'hectares par an (Lanly \& Clément, 1979).

Les forêts qui nous intéressent entreise dans le cadre plus restreint de la forêt tropicale humide - ou forêt hygrophile - qui représente tout de même 750 millions d'hectares dans le monde et pour laquelle tous ces problèmes d'exploitation, de déforestation et de régénération, bien qu'ils soient moins cruciaux, existent néanmoins.

La régénération de ces forêts est évidemment indispensable et pose des problèmes qui ont souvent opposé deux écoles allant même jusqu'à créer un "schisme » entre elles (TAYLOR, 1954), l'une proposant la régénération artificielle, maintenant la plus souvent utilisée en milieu tropical, l'autre défendant la cause de la régénération naturelle elle seule permettant, entre autres, la conservation d'une certaine diversité en espèces dans les forêts tropicales.

Dans cet article, nous abordons le problème de la régénération naturelle de la forêt tropicale humide de la Guadeloupe. Cette question était posée par l'Aménagement de la forêt de la Guadeloupe dont l'arrêté daté du 30-3-1979, stipule que la $4^{\mathrm{e}}$ série, dite d'Essences locales, sera traitée en futaie irrégulière d'essences locales et que pendant une durée de 12 ans des opérations expérimentales de régénération seront effectuées sur 32 ha, le surplus de la série étant laissé au repos.

C'est alors à la demande de l'Office National des Forêts et en collaboration étroite avec cet organisme que nous avons mis en place des dispositifs expérimentaux d'étude de la régénération naturelle des forêts concernées par l'aménagement forestier. 
Dans ce premier article nous présentons les motivations et le cadre de cette étude. Dans un premier chapitre bibliographique nous faisons une analyse des différentes études entreprises dans ce domaine et des techniques mises au point pour favoriser la régénération naturelle en faisant ressortir le degré d'intervention humaine. Ensuite nous nous attardons un peu sur la forêt hygrophile de Guadeloupe ce qui permet de mieux comprendre le choix qui a été fait de s'orienter vers la régénération naturelle de cette forêt plutôt que vers sa transformation en plantations monospécifiques. Enfin, nous décrivons la méthode de régénération naturelle que nous avons commencé à employer ainsi que le dispositif expérimental mis en place pour étudier les différentes phases de cette régénération.

Dans le prochain article, nous nous intéresserons particulièrement aux conséquences des coupes d'ensemencement sur l'installation et la croissance des semis avant que n'aient lieu les premières coupes secondaires, c'est-à-dire pendant les trois ou quatre années suivant la mise en régénération et nous présenterons les premiers résultats concrets déjà obtenus.

\section{Analyse bibliographique}

Les recherches sur la sylviculture des forêts tropicales ne datent pas d'aujourd'hui. Déjà en 1928, STEvenson se préoccupait de «l'aménagement des forêts d'acajou dans le Honduras britannique » et à peu près en même temps, Aubreville (1929) se demandait «Comment constituer une forêt tropicale de rapport » dans laquelle les peuplements issus de régénération naturelle auraient eu une large place.

En 1931, on prévoit pour l'année suivante et avec beaucoup d'optimisme, «l'aménagement des forêts les plus accessibles » de la Guadeloupe (GrEBERT, 1931). Cet aménagement a finalement et fort heureusement vu le jour, nous venons de le voir, près de 50 ans plus tard.

A peu près à cette époque, LAVAUden (1934-1935) se penche sur le passé de la Forêt Equatoriale Africaine et s'inquiète de son avenir.

On retrouve les préoccupations des forestiers français et leurs expérimentations en matière de régénération naturelle lors de la première Conférence forestière Interafricaine à Abidjan en 1951 (BellouARd, 1951). A la même époque, leurs collègues belges ont les mêmes préoccupations au Congo (Maudoux, 1954; WILTEN, 1955 ; DonIs, 1956).

Mais il faut bien le dire, malgré leur enthousiasme, ces forestiers et ces chercheurs n'ont pas réussi à modifier les méthodes de gestion et d'exploitation de la forêt tropicale et leur grande expérience, les circonstances politiques aidant, s'est mal transmise aux générations suivantes.

Au cours des dix dernières années, à l'initiative de la F.A.O. puis de l'I.U.F.R.O. des expériences ont été mises en place en Malaisie et en Thaïlande puis par le C.T.F.T. en Côte-d'Ivoire, en République Centrafricaine et en Guyane Française pour mesurer l'effet des coupes commerciales plus ou moins intensives sur l'accroissment du peuplement restant (ROLLET, 1983). Il est toutefois difficile de ranger ces méthodes pour lesquelles l'exploitation forestière constitue le traitement sylvicole essentiel parmi les 
méthodes de régénération naturelle puisque d'une part on enlève dès le début les principaux semenciers et que d'autre part on ne se préoccupe pas des semis induits par les coupes.

On peut cependant faire un bilan, certainement très incomplet, des différentes études et des différentes méthodes que l'on a tenté de mettre au point pour favoriser, assister ou provoquer la régénération naturelle des forêts tropicales. Pour ce bilan nous avons légèrement débordé du cadre des forêts tropicales humides de moyenne altitude, qui est celui de notre étude. Ceci nous permet de voir que quelque soit la méthode de régénération envisagée - nous les avons classées d'après la nature des interventions humaines lors du processus de régénération - il y a une grande différence entre les régions à saison sèche marquée avec régénération préexistante d'essences commerciales peu abondante et difficile à provoquer et les régions à saison sèche très peu marquée et régénération préexistante abondante et vigoureuse.

\subsection{La régénération naturelle non provoquée}

De nombreux auteurs se sont penchés sur le problème de la reconstitution des forêts primaires tropicales. C'est toute une dynamique qu'il s'agit d'étudier et, même si « une étude complète dépasse les limites de travail d'une vie humaine 》 (WEBB et $a l ., 1972)$, de nombreuses observations ont déjà été faites.

La plupart des études faites quant à la structure et la composition de la forêt tropicale montrent que nous avons à faire à une véritable «mosaïque » (RICHARds, 1952) en éternelle évolution. Des successions d'espèces sont toujours observables et même si l'étude d'une assez grande surface montre un caractère relativement stable, toute une dynamique de transformation de peuplements s'y déroule chaque jour.

Le mécanisme qui entretient cette régénération fait presque toujours appel à la présence de clairières ou «gaps» (WHITMORE, 1978) souvent provoquées par la présence de «chablis» (Oldeman, 1972), qui déclenchent l'installation des espèces héliophiles et favorisent la croissance des espèces sciaphiles presque toujours préexistantes (Rol.et, 1983). Des études ont été faites au sein de ces trouées naturelles et la séparation entre «small gaps» et "large gaps» (Ashron, 1978) se révèle déjà nécessaire. En effet, le comportement des différentes essences rencontrées (essences de lumière, essences d'ombre) jouera directement sur leur présence ou non à l'intérieur de ces clairières plus ou moins grandes.

Par ailleurs, il faudra distinguer les différentes phases pouvant se succéder dans la reconstitution de ces trouées naturelles, phases pionnières et phases secondaires, «dynamic phase» et «homeostatic phase » (Geollegue \& Huc, 1979).

De nombreuses études sur ces successions ont d'ailleurs été faites notamment en Indonésie et Malaisie dans les peuplements de diptérocarpacées (Fox, 1971) mais aussi en Afrique dans des peuplements riches en méliacées (ALEXANDre, 1977) et en Amérique du Sud (Rollet, 1969).

Dans tous les cas, ces études de régénération naturelle «ont un double intérêt : elles permettent de comprendre les mécanismes de transformation des compositions floristiques de forêt dense, et elles sont d'autre part à la base des problèmes pratiques de mise en production des peuplements $\gg$ (RolLET, 1969). 
En effet, la simple observation des taches de régénération naturelle peut déboucher par la suite sur des opérations sylvicoles commandées justement par la présence de ces préexistants. Bien que se basant sur des régénérations déjà acquises, ces méthodes seront classées dans le deuxième paragraphe concernant les régénérations naturelles provoquées ou assistées.

La composition même de ces taches de régénération peut avoir un lien très lointain avec le peuplement en place (Aubreville, 1938) ou, au contraire, peut être relativement significative du peuplement principal (MAZUERA, 1979) mais les différences s'atténuent généralement avec l'ancienneté de la régénération observée.

Malgré tout, et ce sera souvent le cas, les clairières existantes qui ne peuvent être contrôlées entretiennent une régénération vraiment aléatoire sur laquelle le sylviculteur est quelque peu impuissant.

Ainsi, si certaines essences ont une meilleure croissance dans les petites clairières de chablis tel Turraeanthus africana Méliacées, en Afrique (AlEXANDre, 1977), dans la plupart des cas, c'est une insuffisance de lumière qui est à déplorer provoquant une absence quasi totale d'espèces les plus intéressantes mais aussi les plus exigeantes telle Cedrela odorata au Venezuela (PETrT, 1969).

C'est pourquoi, malgré tout l'intérêt que présentent ces études sur la régénération naturelle non provoquée ou études des préexistants, on se doit de s'intéresser principalement aux différentes méthodes sylvicoles permettant de contrôler cette régénération. D'autant que "nous pouvons affirmer qu'il ne faut pas compter sur la nature seule pour régénérer la forêt » (САTiNot, 1965), si par ailleurs l'homme l'a trop perturbée.

\subsection{La régénération naturelle assistée}

Elle est toujours basée sur l'étude des préexistants. Des méthodes statistiques permettant leur dénombrement ont été largement décrites telles que le «linear regeneration sampling 》(BARNARD, 1950) ou le « Milliacre survey» (DouAY, 1954). Suivant les résultats obtenus, les auteurs admettent ou non d'entreprendre des travaux sylvicoles dans les parcelles inventoriées. Nous citerons par exemple les travaux d'amélioration des peuplements d'okoumé (Aucoumea klaineana) au Gabon (LEROY Deval, 1976) ainsi que les différentes méthodes utilisées en Malaisie basées sur l'observation d'un minimum de 2500 semis par hectare avant d'entreprendre des travaux d'entretien et d'empoisonnement du couvert : "Malayan Uniform System » (WYATT-SMITH, 1963).

En réalité, ce genre de méthodes utilisées sur de plus ou moins grandes surfaces correspond à une sylviculture de type jardinage, les interventions consistant en des dégagements de semis en même temps qu'en une coupe permettant la croissance des taches observées (BELL, 1971).

Les bons résultats observés sont en réalité «le fait de forêts naturellement riches en quelques espèces, principalement d'espèces de lumière dont les jeunes plants tolèrent l'ombre dans leur jeunesse » (СATINOT, 1974).

Si, en pratique, le seul moyen d'accroitre directement la densité des semis d'essences de valeur est de faire coïncider les opérations sylvicoles avec une chute abondante de semences d'essences précieuses (SYNNOT \& KEMP, 1976), le problème 
de la fréquence des inventaires des préexistants reste posé ou alors, des observations périodiques de la fructification doivent-elles être faites dans ces forêts? D'autant plus que si le nombre de semis lors des années de fructification est important, peu d'entre eux survivent jusqu'à l'année de fructification suivante, excepté dans les clairières (BuRgEss, 1968).

On peut tout de même envisager de favoriser ou de provoquer cette régénération d'une façon plus systématique et c'est l'objet de notre dernier paragraphe.

\subsection{La régénération naturelle provoquée}

Les méthodes peuvent aller depuis le jardinage à petite échelle jusqu'aux coupes rases d'exploitation forestière. Cependant, il s'est avéré rapidement nécessaire de conserver une certaine ambiance forestière. C'est le but proposé par le Tropical Shelterwood System (Rosevear \& Lancaster, 1953) utilisé très tôt en Afrique, tout d'abord dans les colonies anglaises - Nigéria - puis essayé dans un peu tous les pays. L'opération peut être décrite ainsi (CATINOT, 1965) :

$1^{\mathrm{re}}$ année : Nettoiement des parcelles : coupe des lianes et jeunes brins.

2" annće : Empoisonnement des arbres sans intérêt.

$3^{\circ}$ année : Dégagements de semis.

4" année : Dégagements de semis. Contrôle de la régénération.

$5^{\circ}$ année : Dégagements de semis.

$6^{\prime \prime}$ année : Exploitation.

$7^{\mathrm{K}}$ année : Dégagement, dépressage.

$11^{\circ}$ année : Dégagement, dépressage.

$16^{\circ}$ année : Eclaircies.

$21^{*}$ année : Coupe définitive.

Cette technique, inspirée des méthodes de pays tempérés permet la conservation pendant quelques années de ce nécessaire couvert forestier mais aussi l'apparition progressive d'une quantité de lumière suffisante au sol.

Elle a été appliquée avec réussite à Trinidad sur une dizaine de milliers d'hectares (Duplaquet, 1960) bien que quelquefois des compléments par plantation aient été nécessaires.

Malgré tout, les opposants à cette méthode furent nombreux. Au départ, l'un des principaux problèmes a été le coût des opérations (Rosevear \& LANCASTer, 1953). Certainement aussi quelques difficultés de gestion apparaissaient tant le nombre d'hectares devant être régénérés ainsi était grand et tant le suivi devait être important. Ainsi, des milliers d'hectares ont dû être abandonnés, au Ghana par exemple, où une méthode plus souple de type jardinatoire (Nwoвоsнi, 1976) : la "gestion sélective » apparaissait (exploitation sélective et dégagement de semis tous les 15 ans conservant toujours une bonne répartition des semenciers). L'orientation vers des coupes jardinatoires s'est fait jour aussi, pour les mêmes raisons, au Surinam (Schulz, 1967) où on déplorait de plus l'envahissement herbacé trop important dû aux coupes pourtant nécessaires pour la croissance des semis d'essences précieuses. Cet envahissement sera l'un des facteurs déterminant qui influe directement sur le coût et la réussite de ces opérations. Certains auteurs pensent pouvoir réduire quelque peu ce type d'intervention en faisant, par exemple, des dégagements par bandes (Schulz, 1967). 
Notons enfin que si parfois des observations de régénération après coupe rase d'exploitation semblent être jugées positives, comme en Dominique (BELL, 1976), il faudra se méfier a priori d'une part d'une ouverture trop grande du couvert qui risque de transformer complètement la composition floristique future — étude du recrû après coupe papetière en Guyane - (DE Foresta, Prevost, 1981), mais aussi des marques laissées par les exploitations mécanisées laissant sur place de véritables «ćcotypes d'exploitation» (Fox, 1972) où une absence totale de régénération risque de se faire sentir.

En résumé de cette analyse bibliographique sommaire, nous pouvons dire que ces problèmes de régénération naturelle des forêts tropicales sont encore mal résolus et qu'il est nécessaire de continuer à observer et à expérimenter. En effet, l'application de la régénération naturelle est devenue trop rare en milieu tropical en raison d'un «manque de prise de responsabilité vis-à-vis des forêts tropicales et un manque de connaissances sylvoécologiques 》(LAMPrechT, 1976). C'est pourquoi il nous semble indispensable d'initier ou de poursuivre des études dans ce domaine et c'est aussi dans cette optique que nous avons entrepris nos études sur la régénération naturelle de la forêt tropicale humide de Guadeloupe.

Le but de nos expériences est d'obtenir après régénération un nouveau peuplement plus riche en essences précieuses. Pour cela, nous avons utilisé une méthode voisine de la régénération naturelle par coupes progressives et donc du Tropical shelterwood system, en pratiquant des coupes d'ensemencement d'intensité variable et des coupes secondaires variables en nombre et en fréquence.

Ceci nous permettra, en plus, d'obtenir le maximum d'informations sur le tempérament de ces différentes espèces qui, pour l'instant, est à peu près inconnu et de choisir les méthodes sylvicoles à employer pour favoriser telle ou telle espèce.

\section{La forêt hygrophile de Guadeloupe}

\subsection{Sa place dans la forêt guadeloupéenne}

Les conditions particulières de relief et de topographie des deux îles qui constituent la Guadeloupe créent de grandes différences climatiques, notamment en ce qui concerne les précipitations. Ceci explique la diversité des formations végétales rencontrées sur les 1500 kilomètres carrés de la Guadeloupe (Basse Terre et Grande Terre uniquement). Les formations forestières définies par STEHLE (1946) ont été regroupées en trois types liés à la pluviométrie : xérophile, mésophile et hygrophile ainsi que le montre la figure 1.

La forêt xérophile est essentiellement rencontrée dans la Côte-sous-le-Vent de la Basse Terre et dans la Grande Terre. Située à une altitude inférieure à $250 \mathrm{~m}$ et bordant le littoral, elle reçoit annuellement entre 1000 et $2000 \mathrm{~mm}$ d'eau. Cette forêt a été fortement perturbée par la présence humaine. De nombreuses cueillettes ainsi que des défrichements importants l'ont souvent transformée en friches ou en terres agricoles à vocation agrumicole. 


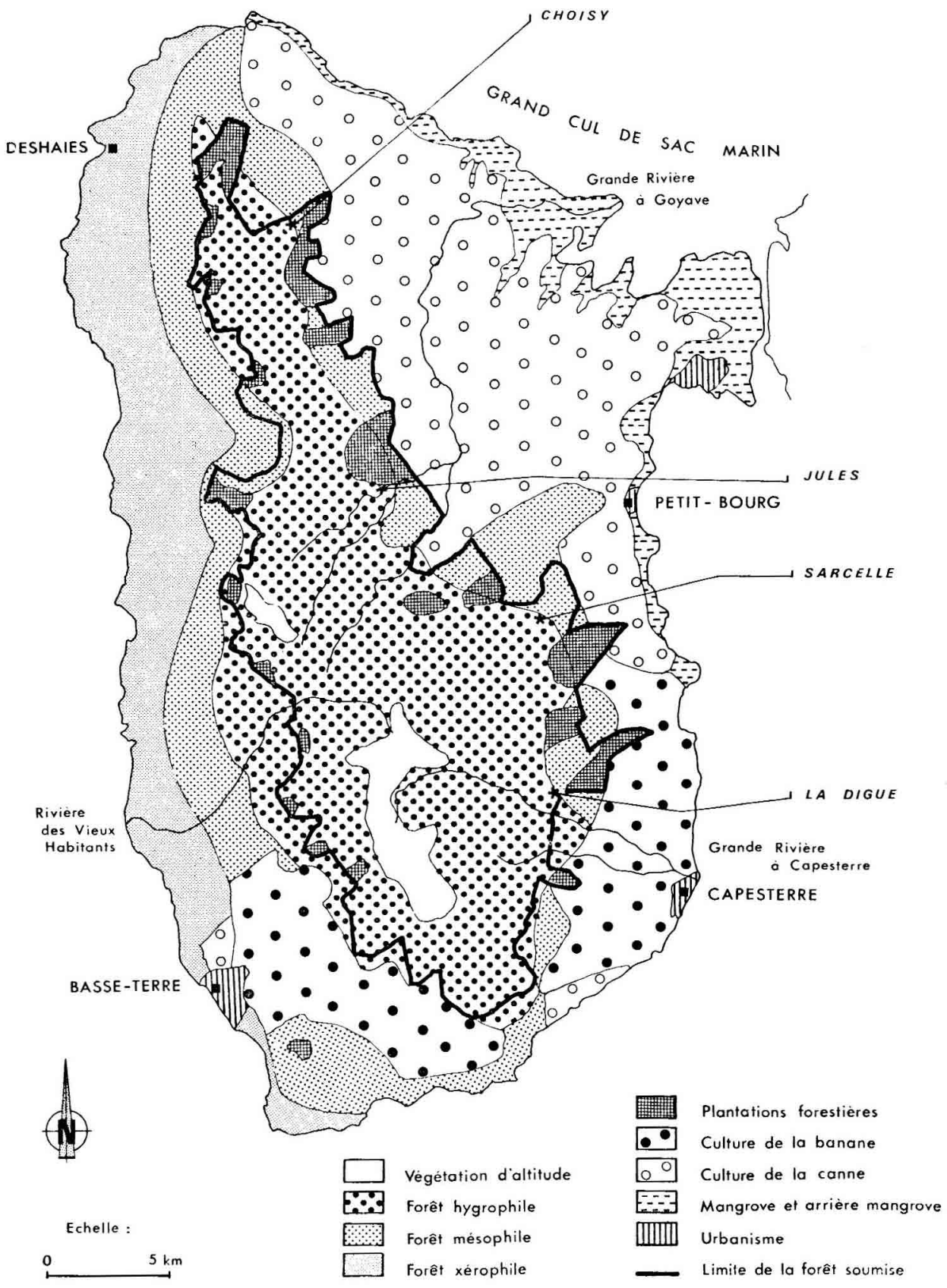

FIG. 1

Carte d'occupation des sols et des formations forestières de la Guadeloupe (seule l'île de la Basse Terre est représentée) réalisée d'après l'aménagement forestier. Map of farm land and forest type of Guadeloupe (French West Indies). Only the Basse Terre island has been drawn (Source : Forest management plan). 
La forêt mésophile est représentée aussi bien en Côte-au-Vent qu'en Côte-sousle-Vent de la Basse Terre. Son aire est simplement limitée par les isohyètes $2000 \mathrm{~mm}$ et $3000 \mathrm{~mm}$. Cette forêt a subi de nombreuses dégradations par cueillette des bois de valeur et c'est dans cette formation que se sont installées les plantations de banane et de canne à sucre.

La forêt hygrophile est le type de formation actuellement le plus représenté. Elle est située à des altitudes variant de $400 \mathrm{~m}$ à $1000 \mathrm{~m}$ et reçoit en moyenne des précipitations de 3000 à $6000 \mathrm{~mm}$.

Au-dessus de $1000 \mathrm{~m}$, cette forêt devient rabougrie et laisse place à des savanes d'altitude, cet ensemble jouant un rôle important dans la protection des sols.

Il est difficile d'avoir une estimation précise des superficies occupées par les formations forestières et elles varient entre 60000 ha et 90000 ha selon les sources documentaires.

La forêt soumise n'occupe que 28000 ha situés dans le massif montagneux de la Basse Terre (voir fig. 1). Elle a fait l'objet d'un aménagement forestier valable pour la période 1979-1990 et a été divisée à cette occasion en quatre séries d'aménagement.

La première série (15000 ha), hors cadre, est une série de protection constituéc par les forêts rabougries et les savanes d'altitude.

La deuxième série ( 8000 ha), dite de transformation, occupe les zones dégradées de la forêt mésophile et est en cours d'enrichissement grâce à des plantations d'acajou du Honduras (Swietenia macrophylla).

La troisième série ( $500 \mathrm{ha}$ ), dite de laurier rose, occupe les parties hautes de la forêt hygrophile proches de la Soufrière et est consacrée à la sylviculture de cette espèce (Podocarpus coriaceus).

La quatrième série (4 400 ha), dite d'essences locales, occupe la majeure partie de la forêt hygrophile.

Cette quatrième série est en repos pendant la durée du présent aménagement. Seules y ont été prévues les expérimentations sur la régénération naturelle objet du présent article.

\subsection{Composition de la forêt hygrophile}

La forêt hygrophile est caractérisée climatiquement, outre ses précipitations annuelles de 3000 à $6000 \mathrm{~mm}$ réparties sur plus de 300 jours, par une humidité toujours supéricure à 75 p. 100 et des températures moyennes variant entre $22{ }^{\circ} \mathrm{C}$ et $27{ }^{\circ} \mathrm{C}$.

La forêt hygrophile, formation la moins perturbée en raison de la difficulté d'accès, présente une structure complexe depuis les strates herbacées, lianoïdes, épiphytes, jusqu'aux strates arborescentes dominantes. Cette stratification complexe permet la présence d'espèces nombreuses dont les plus fréquentes et les plus importantes sont : 
pour la strate arborescente dominante :

- gommier blanc

- bois rouge carapate

- corossolier montagne

- mapou baril

- châtaignier grande feuille

- acomat boucan

— autres châtaigniers
Dacryodes excelsa

Amanoa caribaea

Guatteria caribaea

Sterculia caribaea

Sloanea massoni

Sloanea caribaea

Sloanea truncata, S. Berteriana

pour la strate arborescente moyenne :

- marbri

- acajou blanc

- cotelette noire

- mauricifs

— caïmitiers, balatas
Richeria grandis

Simaruba amara

Tapura latifolia

Byrsonima sp.

Pouteria sp.

pour la strate arborescente inférieure :

- guépois

- cassant

pour la strate arbustive :

- bois cotelette

- arbustes divers appartenant aux genres suivants :
Myrcia leptoclada

Rudgea citrifolia

Cette liste est évidemment très incomplète et on observe de grandes variations géographiques dans la composition botanique de la forêt. Seuls quelques auteurs : Duss (1897), Stehle (1945), Beard (1949) ei Dulau (1956) se sont penchés sur ce problème mais n'ont pas abouti à une véritable zonation écologique des différentes espèces de la forêt hygrophile.

L'inventaire statistique réalisé en 1974, préalablement à l'aménagement forestier, donne des informations complémentaires sur la quatrième série. Le nombre de tiges de diamètre supérieur à $12,5 \mathrm{~cm}$ est de 646 par hectare. Ceci correspond à une surface terrière de $39,5 \mathrm{~m}^{2} / \mathrm{ha}$. Les espèces principales, dites précieuses, et reconnues comme telles dans l'aménagement représentent 48 p. 100 du nombre total de tiges (dont 20,7 p. 100 pour le gommier, 12,6 p. 100 pour le bois rouge, 8,5 p. 100 pour le marbri et 1,8 p. 100 pour l'acajou blanc) et 64 p. 100 de la surface terrière.

Une cstimation des volumes sur pied donne pour les arbres de diamètre supérieur à $30 \mathrm{~cm}$, un total de $236 \mathrm{~m}^{3}$ par hectare dont 148 pour les essences principales $\left(67,3 \mathrm{~m}^{3}\right.$ pour le gommier, $62 \mathrm{~m}^{3}$ pour le bois rouge et moins de $10 \mathrm{~m}^{3}$ pour chacune des autres espèces) et 88 pour les espèces diverses.

Cette richesse en essences principales, la prédominance numérique de deux de ces espèces, le gommier et le bois rouge, ainsi que l'importance du volume exploitable font de cette forêt un cas particulier des forêts tropicales où en général — c'est 
le cas de la majorité des forêts africaines et de la forêt amazonienne - aucune espèce précieuse n'est prédominante et où les volumes exploitables sont faibles.

Cette situation justifie l'importance que l'on attache en Guadeloupe à la régénération naturelle de cette forêt et, par cette méthode, à l'augmentation du pourcentage d'essences principales.

\subsection{Principales espèces pricieuses de la forêt hygrophile}

L'Aménagement n'a retenu qu'une dizaine d'essences principales susceptibles d'utilisation en raison de leur qualité, leur tradition d'usage et leur fréquence dans les peuplements. En fait, d'autres essences sont utilisables et GreBERT (1931) indique l'usage d'une vingtaine d'espèces principalement pour le charronnage et la construction traditionnelle, les qualités recherchées étant la dureté, l'imputrescibilité et la résistance aux termites.

En fait, toutes ces espèces sont encore mal connues en dépit des travaux de Duss (1897), Stehle (1936, 1937), Little et al. (1964, 1974) et Fournet (1978) dans le domaine botanique et ceux de LoNGwood (1962) sur les caractéristiques technologiques des bois. En particulier, on n'a pratiquement pas de renseignements sur la biologie de la reproduction et les lois de croisement (espèces autogames ou hétérogames) des essences principales dont nous étudions la régénération naturelle.

Le gommier blanc (Dacryodes excelsa Vahl. Burseraceae) est très répandu en forêt hygrophile. Il occupe une strate altitudinale allant de $350 \mathrm{~m}$ à $950 \mathrm{~m}$. Au niveau inférieur il est devenu rare, semble-t-il à cause des exploitations et défrichements abusifs. C'est un arbre très grand atteignant $30 \mathrm{~m}$ de hauteur. Le fût est très élancé, sans branches, le diamètre à la base pouvant dépasser un mètre. La base du tronc est un peu élargie et présente quelquefois de courts contreforts.

Le bois de cœur est brun pâle puis brun rosé après séchage.

L'aubier, rarement très épais, apparaît gris clair. La texture est fine. Le bois est très chargé en silice. La densité est de 0,64 après séchage à l'air et de 0,47 à l'état vert. Le séchage se fait en trois mois minimum avec un retrait radial de 4,1 p. 100 et tangentiel de 6,4 p. 100 . Il est utilisé principalement pour la menuiserie, la charpente et la caisserie. Une utilisation plus ancienne concernait la fabrication de canots.

Le bois rouge carapate (Amanoa caribaea $\mathrm{Kr}$. et Urb. Euphorbiaceae) est endémique de la Guadeloupe et de la Dominique et se trouve assez fréquemment en forêt hygrophile à des altitudes allant de 300 à $850 \mathrm{~m}$.

C'est un grand arbre pouvant atteindre $30 \mathrm{~m}$ avec un fût sans branche sur plus de $15 \mathrm{~m}$. Le tronc est de couleur rouge sombre avec des aspérités blanchâtres. La base du tronc est généralement anfractueuse avec des contreforts prononcés. Le bois est particulièrement dur et imputrescible et résiste bien aux termites.

L'aubier assez important est brun rouge, le cour est marron. Le retrait volumétrique, toujours élevé, varie de 17,7 à 19,4 p. 100 . Il est utilisé principalement pour la charpente et la construction. 
Le marbri (Richeria grandis Vahl. Euphorbiaceae) est essentiellement cantonné dans la forêt hygrophile bien qu'on puisse le trouver dans la forêt mésophile plus sèche. On le retrouve ainsi à des altitudes allant de $150 \mathrm{~m}$ à $1000 \mathrm{~m}$. L'arbre est de taille moyenne, généralement inférieure à $20 \mathrm{~m}$. Le tronc est droit et élancé. Le bois est résistant aux termites. Sa densité est de 0,74 à 12 p. 100 d'humidité. Il est utilisé essentiellement pour la construction, mais peut aussi servir en ébénisterie.

L'acajou blanc (Simaruba amara Aubl. Simarubaceae) est présent dans tout lare Antillais ainsi qu'en Guyane et en Amazonie. Il est fréquent dans les zones inférieures de la forêt hygrophile ainsi qu'en forêt mésophile. On le trouve entre 250 et $700 \mathrm{~m}$ d'altitude. L'arbre est assez grand et élancé. Le bois est blanc et il est difficile de distinguer le cœur de l'aubier. C'est un bois très tendre qui résiste aux termites. Sa densité est de 0,44 à l'état vert et de 0,38 après séchage à l'air. Le retrait volumétrique est faible : 8 p. 100 mais sensible aux variations hygrométriques. Il est principalement exploité à des fins de boiserie et caisserie mais peut être utilisé en menuiserie.

Le palétuvier jaune (Symphonia globulifera L. Clusiaceae) est un arbre de bas de versants inondés et présente des racines échasses caractéristiques. Il donne un excellent bois utilisć aussi bien pour la charpente, la caisserie que la menuiserie fine.

Le mauricif patagon (Byrsonima coriacea, Malpighiaceae) est un arbre moyen occupant la partie inférieure de la forêt hygrophile. Il est plus fréquent en forêt mésophile. Son bois est utilisé pour la charpente.

Bien que les forestiers locaux fassent la différence entre le mauricif patagon et le mauricif bois-charbon, il est difficile botaniquement de faire la séparation entre ces deux appellations locales et les quatre espèces botaniques concernées $B$. coriacea, $B$. laevigata, $B$. martinicensis ou $B$. trinitensis.

Le résolu (Chimarrhis cymosa Jacq. Rubiaceae) rencontré principalement le long des cours d'eau fournit un bois utilisé en menuiserie et ébénisterie.

Le bois doux chypre (Phoebe elongata Vahl. Lauraceae) est un arbre moyen situé en partie basse de la forêt hygrophile. Son bois, très prisé, est utilisé pour l'ébénisterie fine. Là encore cette appellation locale est susceptible d'englober d'autres espèces de la famille des Lauracées car les identifications faites par les agents forestiers ne sont pas toujours concordantes.

\section{Dispositif d'étude de la regénération naturelle}

\subsection{Le protocole expérimental}

Comme nous l'avons déjà dit en conclusion de notre analyse bibliographique, nous avons utilisé une méthode voisine de la régénération naturelle par coupes progressives dont dérivent en fait les méthodes utilisées en forêt tropicale et connues principalement sous le nom de "Tropical shelterwood system» et de «Malayan shelterwood system $»$. 
Comme nous n'avions aucune information a priori sur le tempérament des différentes espèces à régénérer, nous avons imaginé plusieurs combinaisons entre l'intensité de la coupe d'ensemencement, le nombre, l'intensité et la fréquence des coupes secondaires afin de créer des conditions écologiques aussi différentes que possible tout en restant dans des limites raisonnables. En effet, une mise en lumière trop rapide entraîne un recrû herbacé et arbustif très abondant et conduit à une augmentation considérable des dégagements. Inversement, une mise en lumière trop prudente peut rallonger inutilement la durée de la régénération ou même la mettre en danger.

Toutefois, nous pensons que les conditions écologiques créées au niveau du sol, par les coupes les plus fortes, favoriseront la régénération des essences de lumière, tandis que celles créces par les coupes les plus faibles favoriseront les essences d'ombre.

Pour cela, nous avons choisi quatre traitements sylvicoles, deux débutant par une coupe d'ensemencement dite «faible» et deux débutant par une coupe d'ensemencement dite «forte». Les deux parcelles correspondant à chaque type de coupe diffèrent ensuite par la durée de la phase de régénération, c'est-à-dire par le nombre et la fréquence des coupes secondaires ainsi que l'indique le tableau 1 .

TABLEAU 1

Calendrier des différentes coupes à effectuer dans les dispositifs de régénération naturelle.

Planning of the different fellings in the experimental designs on natural regeneration.

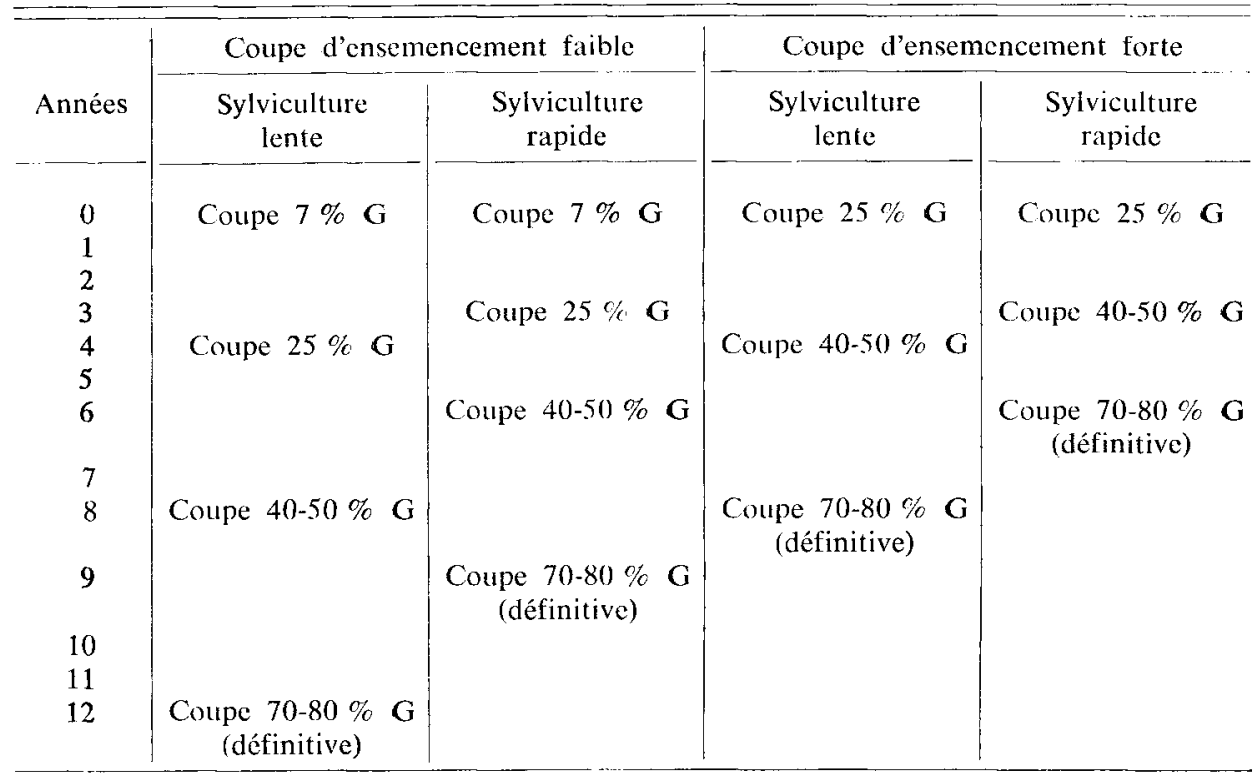

Remarque : une coupe à $x \%$ de $\mathbf{G}$ est telle que, après coupe, le pourcentage de surface terrière enlevé depuis le début de l'expérience est égal à $x$. 
Des travaux de dégagement des semis ont été initialement prévus de manière un peu arbitraire, mais nous pensons, au vu des premiers résultats, qu'il vaut mieux les prévoir au fur et à mesure des besoins, en essayant de trouver un compromis entre un nombre de dégagements trop important et la concurrence que peuvent raisonnablement supporter les semis.

Au vu des premiers résultats, on peut déjà dire que dans les coupes fortes des dégagements bisannuels sont suffisants et que dans les coupes faibles on peut aller jusqu'aux premières coupes secondaires sans dégagement.

\subsection{Emplacement et caractéristiques des dispositifs expérimentaux}

Chaque dispositif expérimental est constitué de quatre parcelles de régénération de 2 hectares chacune et d'une parcelle témoin d'un hectare. Les parcelles sont sćparées les unes des autres par des bandes tampons de 40 à $50 \mathrm{~m}$ et on a ménagé, tout autour du périmètre expérimental, une bande tampon de même largeur, ce qui porte la superíicic d'un dispositif expérimental à environ 16 hectares.

Nous avons installé au total quatre dispositifs expérimentaux en quatre endroits

\section{TABlenu 2}

Caractéristiques dendrométriques des parcelles expérimentales avant et après la coupe d'ensemencement.

\begin{tabular}{|c|c|c|c|c|c|c|c|c|}
\hline \multirow[b]{2}{*}{ Parcelle } & \multicolumn{5}{|c|}{ Jules } & \multicolumn{3}{|r|}{ Sarcell } \\
\hline & 1 & 2 & 3 & 4 & moy. & 1 & 2 & 3 \\
\hline Type de coupe & forte & faible & forte & faible & & forte & faible & forte \\
\hline $\mathrm{N}$ initial/ha & 623,5 & 627,5 & 737 & 770 & 689,5 & 549,5 & 652 & 616,5 \\
\hline $\mathrm{N}$ final $/$ ha $\ldots \ldots \ldots \ldots \ldots \ldots \ldots$ & 470,5 & 573,5 & 552 & 697 & & 401,5 & 596 & 431 \\
\hline$\% \mathrm{~N}$ enlevé $\ldots \ldots \ldots \ldots \ldots \ldots \ldots$ & 24,5 & 8,6 & 25,1 & 9,5 & & 26,9 & 10,0 & 30,1 \\
\hline$\frac{N \text { divers enlevé }}{N \text { total enlevé }} \times 100 \ldots \ldots \ldots \ldots$ & 93 & 94 & 91 & 92 & & 99 & 98 & 96 \\
\hline $\mathrm{G}$ initial $\mathrm{m}^{2} / \mathrm{ha} \ldots \ldots \ldots \ldots \ldots$ & 41,0 & 35,8 & 39,3 & 45,0 & 40,3 & 38,1 & 45,9 & 42,6 \\
\hline $\mathrm{G}$ final $\mathrm{m}^{2} / \mathrm{ha}$ & 35,5 & 34,1 & 33,4 & 42,4 & & 29,3 & 44,2 & 33,1 \\
\hline$\% \mathrm{G}$ enlevé $\ldots . .$. & 13,4 & 4,7 & 15,0 & 5,8 & & 23,1 & 3,7 & 22,3 \\
\hline$\% \mathrm{~N}$ précieux initial & 41,7 & 38,3 & 43,8 & 49,6 & 43,7 & 47,4 & 44 & 49,6 \\
\hline$\% \mathrm{~N}$ précieux final ... & 53,2 & 41,3 & 55,5 & 53,9 & & 64,5 & 48,7 & 69,4 \\
\hline$\%$ G précieux initial $\ldots \ldots \ldots$ & 58,3 & 57,5 & 59,8 & 62,3 & 59,6 & 66,1 & 62,8 & 68,8 \\
\hline$\%$ G précicux final $\ldots \ldots \ldots \ldots \ldots$ & 67,3 & 60,4 & 70,4 & 66,5 & & 86,0 & 65,2 & 88,5 \\
\hline$\frac{\mathrm{GB}}{\mathrm{BM}}$ initial $\ldots \ldots \ldots \ldots \ldots \ldots$ & 0,14 & 0,15 & 0,09 & 0,11 & & 0,20 & 0,20 & 0,17 \\
\hline$\frac{\mathrm{GB}}{\mathrm{BM}}$ final & 0,15 & 0,16 & 0,08 & 0,11 & & 0,24 & 0,22 & 0,19 \\
\hline 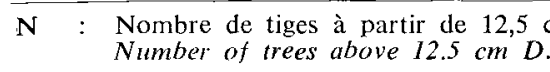 & $\begin{array}{l}\mathrm{cm} \text { de d } \\
. B . H .\end{array}$ & liamètre. & & & & & & \\
\hline $\begin{aligned} \mathrm{G}: \text { Surface terrière des } \mathrm{N} \text { tiges. } \\
\text { Basal area. }\end{aligned}$ & & & & & & & & \\
\hline
\end{tabular}


différents de la forêt hygrophile. Du nord au sud de la Basse Terre et situés sur la Côte-au-vent on trouve :

- Choisy à $250 \mathrm{~m}$ d'altitude sur la commune de Sainte-Rose,

- Jules à $230 \mathrm{~m}$ d'altitude sur la commune de Petit-Bourg,

- Sarcelle à $270 \mathrm{~m}$ d'altitude sur la commune de Goyave,

- et La Digue à $450 \mathrm{~m}$ d'altitude sur la commune de Capesterre-Belle-Eau.

Les trois premicrs dispositifs sont situés sur des sols ferrallitiques issus du volcanisme ancien. Les précipitations estimées d'après les isohyètes de la carte de LASSERRE (1961) voisinent $3500 \mathrm{~mm}$ par an. Le dernier dispositif, le plus au sud est situé sur des sols à allophanes issus du volcanisme plus récent. Adossé au massif de la Soufrière, il jouit annuellement de précipitations estimées à près de $4500 \mathrm{~mm}$. Chaque parcelle a fait l'objet d'un inventaire en plein à partir de $12,5 \mathrm{~cm}$ de diamètre. Dans tout ce qui suit, les nombres de tiges et surfaces terrières ne concernent que les arbres supérieurs à ce diamètre. On a inventorié séparément chacune des huit espèces principales, toutes les autres ayant été considérées comme "divers».

Le tableau 2 indique la densité, la surface terrière ainsi que le pourcentage d'espèces précieuses et diverses dans chaque parcelle.

Main characteristics of the experimental plots before and after the seed fellings.

\begin{tabular}{|c|c|c|c|c|c|c|c|c|c|c|c|c|}
\hline & & \multicolumn{5}{|c|}{ La Digue } & \multicolumn{5}{|c|}{ Choisy } & $4^{\prime \prime}$ série \\
\hline 4 & moy. & 1. & 2 & 3 & 4 & moy. & 1 & 2 & 3 & 4 & moy. & moy. \\
\hline faible & & forte & faible & faible & forte & & forte & forte & faible & faible & & \\
\hline $\begin{array}{c}631,5 \\
570 \\
9,7\end{array}$ & 615 & $\begin{array}{c}507 \\
348 \\
31,4\end{array}$ & $\begin{array}{l}520 \\
435 \\
16,3\end{array}$ & $\begin{array}{l}557 \\
457 \\
18,0\end{array}$ & $\begin{array}{r}454,5 \\
304,5 \\
33,0\end{array}$ & 510 & $\begin{array}{r}445,5 \\
318,5 \\
28,5\end{array}$ & $\begin{array}{l}606 \\
417 \\
31,2\end{array}$ & $\begin{array}{c}449,5 \\
393 \\
12,6\end{array}$ & $\begin{array}{l}577 \\
490 \\
15,1\end{array}$ & 519,5 & 646 \\
\hline 94 & & 99 & 91 & 93 & 96 & & 99 & 100 & 99 & 99 & & \\
\hline 46,6 & 43,3 & 36,5 & 41,8 & 38,3 & 34,6 & 37,8 & 42,5 & 34,1 & 34,1 & 37,6 & 37,1 & 39,5 \\
\hline 44,8 & & 28,3 & 38,8 & 35,9 & 27,1 & & 35,1 & 26,2 & 31,9 & 35,2 & & \\
\hline 3,9 & & 22,5 & 7,3 & 6,3 & 21,8 & & 17,4 & 23,2 & 6,5 & 6,4 & & \\
\hline 53,4 & 48,6 & 30,7 & 44,3 & 41,2 & 31,6 & 37,2 & 35,7 & 16,2 & 14,9 & 16,7 & 20,3 & 48,0 \\
\hline 58,5 & & 44,3 & 51,2 & 486 & 45,3 & & 49,5 & 23,6 & 16,9 & 19,5 & & \\
\hline 71,0 & 67,2 & 50,7 & 64,6 & 61.9 & 57,2 & 58,9 & 55,3 & 34,6 & 302 & 38,8 & 40,6 & 64,0 \\
\hline 73,9 & & 65,4 & 69,6 & 66,0 & 73,1 & & 67,0 & 45 & 32,3 & 41,5 & & \\
\hline 0,24 & & 0,43 & 0,51 & 0,44 & 0,39 & & 0,57 & 0,25 & 0,42 & 0,38 & & \\
\hline 0,24 & & 0,45 & 0,51 & 0,44 & 0,50 & & 0,53 & 0,29 & 0,43 & 0,39 & & \\
\hline
\end{tabular}

GB : Nombre de gros arbres à partir de $52,5 \mathrm{~cm}$ de diamètre. Number of big trees above or equal to $52.5 \mathrm{~cm}$ D.B.H.

$\mathrm{BM}$ : Nombre d'arbres moyens de 32,5 à $52,5 \mathrm{~cm}$ de diamètre. Number of medium trees from $32.5 \mathrm{~cm}$ to $52.5 \mathrm{~cm}$ D.B.H. 
TABLEAU 3

Composition initiale des différentes parcelles expérimentales en espèces précieuses exprimée en pourcentage du nombre de tiges total $(N)$ et de la surface terrière totale $(G)$ de chaque parcelle.

\begin{tabular}{|c|c|c|c|c|c|c|c|c|c|}
\hline & & \multirow{2}{*}{$4^{\circ}$ série } & \multicolumn{5}{|c|}{ Jules } & \multicolumn{2}{|c|}{ Sarcell } \\
\hline & & & 1 & 2 & 3 & 4 & moy. & 1 & 2 \\
\hline Gommier & $\begin{array}{l}\mathbf{N} \\
\mathrm{G}\end{array}$ & 20,7 & $\begin{array}{l}16,6 \\
25,0\end{array}$ & $\begin{array}{l}17,0 \\
26,0\end{array}$ & $\begin{array}{l}18,8 \\
240\end{array}$ & $\begin{array}{l}22,0 \\
25,0\end{array}$ & $\begin{array}{l}20,0 \\
25,0\end{array}$ & $\begin{array}{l}28,5 \\
45,0\end{array}$ & $\begin{array}{l}26,9 \\
43,0\end{array}$ \\
\hline Bois rouge & $\begin{array}{l}N \\
G\end{array}$ & 12,6 & $\begin{array}{l}15,3 \\
27,0\end{array}$ & $\begin{array}{l}14,7 \\
28,0\end{array}$ & $\begin{array}{l}13,2 \\
28,0\end{array}$ & $\begin{array}{l}12,4 \\
29,0\end{array}$ & $\begin{array}{l}14,0 \\
28,0\end{array}$ & $\begin{array}{r}9,6 \\
15,0\end{array}$ & $\begin{array}{r}8,0 \\
15,0\end{array}$ \\
\hline Marbri & $\begin{array}{l}N \\
G\end{array}$ & 8,5 & $\begin{array}{l}5,8 \\
4,0\end{array}$ & $\begin{array}{l}4,5 \\
3,0\end{array}$ & $\begin{array}{l}9,7 \\
6,0\end{array}$ & $\begin{array}{r}11,8 \\
7,0\end{array}$ & $\begin{array}{l}8,0 \\
5,0\end{array}$ & $\begin{array}{l}3,6 \\
2,0\end{array}$ & $\begin{array}{l}4,7 \\
2,0\end{array}$ \\
\hline Acajou blanc & $\begin{array}{l}N \\
G\end{array}$ & 1,8 & $\begin{array}{l}3,1 \\
2,0\end{array}$ & $\begin{array}{l}1,4 \\
1,0\end{array}$ & $\begin{array}{l}0,5 \\
1,0\end{array}$ & $\begin{array}{l}1,3 \\
1,0\end{array}$ & $\begin{array}{l}1,5 \\
1,2\end{array}$ & $\begin{array}{l}2,0 \\
1,0\end{array}$ & $\begin{array}{l}1,8 \\
1,0\end{array}$ \\
\hline
\end{tabular}

La variabilité de ces données est assez grande d'une parcelle à l'autre et d'un dispositif expérimental à l'autre, aussi les différences observées ne sont-elles pas toujours significatives.

Le nombre de tiges par hectare varie de 445 à 770 et doit être rapproché de la moyenne de 646 tiges par hectare pour l'ensemble de la $4^{*}$ série d'aménagement. Ceci peut être interprété notamment grâce aux histogrammes donnant la structure des peuplements pour chacun des 4 dispositifs (fig. 2).

Ces histogrammes ont la même forme que ceux observés dans d'autres forêts tropicales (Rollet, 1969) et sont en tous points identiques à ceux des futaies jardinées des forêts tempérées. Bien qu'en forêt tropicale on soit en présence d'un nombre très élevé d'espèces, on peut voir dans cette similitude de structure une même stratégie d'occupation de l'espace par la cime des différents arbres. Les histogrammes de Jules et Sarcelle font penser à des peuplements rajeunis comportant un nombre important de tiges de petit diamètre (de $12,5 \mathrm{~cm}$ à $17,5 \mathrm{~cm}$ ) et un rapport gros bois sur bois moyen faible résultant d'exploitations plus ou moins anciennes (c'est le cas de Jules) ou de chablis eux aussi plus ou moins anciens (c'est le cas de Sarcelle). La Digue et Choisy peuvent être considérés comme des peuplements plus anciens, voire même vieillis, ayant moins de tiges de petit diamètre et un rapport gros bois sur bois moyen bien supérieur à celui de la moyenne de la $4^{\mathrm{e}}$ série.

Ces différences entre peuplements sont moins visibles pour la surface terrière et il faudrait tenir compte des différences stationnelles de fertilité pour les expliquer: 
Initial composition of the different experimental plots with timber species expressed as percent of the total tree number $(N)$ and of the total basal area (G) of each plot.

\begin{tabular}{|c|c|c|c|c|c|c|c|c|c|c|c|c|}
\hline \multirow[b]{2}{*}{3} & \multirow[b]{2}{*}{4} & \multirow[b]{2}{*}{ moy. } & \multicolumn{5}{|c|}{ La Digue } & \multicolumn{5}{|c|}{ Choisy } \\
\hline & & & 1 & 2 & 3 & 4 & moy. & 1 & 2 & 3 & 4 & moy. \\
\hline 34,5 & 38,0 & 32,0 & 13,2 & 16,4 & 15,0 & 20,4 & 16,0 & 22,1 & 9,5 & 10,2 & 5,5 & 11,0 \\
\hline 50,0 & 54,0 & 48,0 & 32,4 & 31,5 & 34,0 & 46,1 & 35,7 & 36,0 & 22,0 & 32,0 & 17,0 & 27,0 \\
\hline 7,2 & 9,4 & 9,0 & 3.6 & 18,4 & 16,5 & 4,4 & 110 & 8,4 & 3,7 & 00 & 4,9 & 4,0 \\
\hline 11,0 & 13,0 & 130 & 5,7 & 28,6 & 21,7 & 5.2 & 16,0 & 16,0 & 6,0 & 0,0 & 13,0 & 9,0 \\
\hline 3,7 & 1,3 & 30 & 9,3 & 6.2 & 5,8 & 4,3 & 6,0 & 1,9 & 0,4 & 09 & 3,1 & 1,6 \\
\hline 2,0 & 1,0 & 1,7 & 7,4 & 3,2 & 3,8 & 4,2 & 4,6 & 10 & 0,4 & 0,4 & 2.0 & 1,0 \\
\hline 1,3 & 1,6 & 20 & 3,9 & 1.4 & 27 & 1.8 & 2.5 & 1,6 & 0,7 & 02 & 1,1 & 0,9 \\
\hline 1,0 & 1,0 & 10 & 20 & 1,3 & 1,4 & 10 & 1,3 & 1,0 & 1,0 & () 1 & 1,0 & 0,8 \\
\hline
\end{tabular}

La richesse en espèces précieuses varie aussi beaucoup d'un dispositif à l'autre : 48,6 p. 100 du nombre de tiges et 67,2 p. 100 de la surface terrière à Sarcelle contre 20,3 p. 100 et 40,6 p. 100 respectivement pour Choisy, ces chiffres étant de toute façon inférieurs à la moyenne de la $4^{*}$ série, ceci voulant dire que les dispositifs, placés à proximité d'accès et donc situés dans la partie basse de la $4^{\circ}$ série, n'incluent pas les peuplements les plus riches de cette série.

La répartition des 4 espèces les plus fréquentes, dans l'ordre décroissant : le gommier, le bois rouge, le marbri puis l'acajou blanc, est donnée dans le tableau 3. La répartition des essences est très variable. Le gommier représente entre 5,5 p. 100 et 38 p. 100 du nombre de tiges par parcelle, tandis que le bois rouge carapate qui est parfois absent représente au plus 18,4 p. 100 des tiges. Le marbri est plus fréquent dans certains dispositifs que dans d'autres, tandis que l'acajou blanc est toujours présent en petite quantité.

On ne peut pas, pour l'instant, expliquer ces différences de fréquence des espèces qui dépendent à la fois de leurs exigences écologiques et de l'histoire du peuplement. Ceci nécessiterait une étude de l'ensemble du massif forestier.

L'installation des dispositifs s'est étalée entre novembre 1979 et décembre 1981, ainsi que l'indique le tableau 4. Les premiers dégagements de semis ont eu lieu de septembre 1981 à février 1982 dans les deux premiers dispositifs installés, c'est-àdire Jules et Sarcelle, en août 1982 pour La Digue et en juillet 1983 pour Choísy. $\mathrm{Au}$ même moment, on réalisait les deuxièmes dégagements dans les parcelles coupes fortes de Jules et Sarcelle. 
nombre darbres

par hectare.
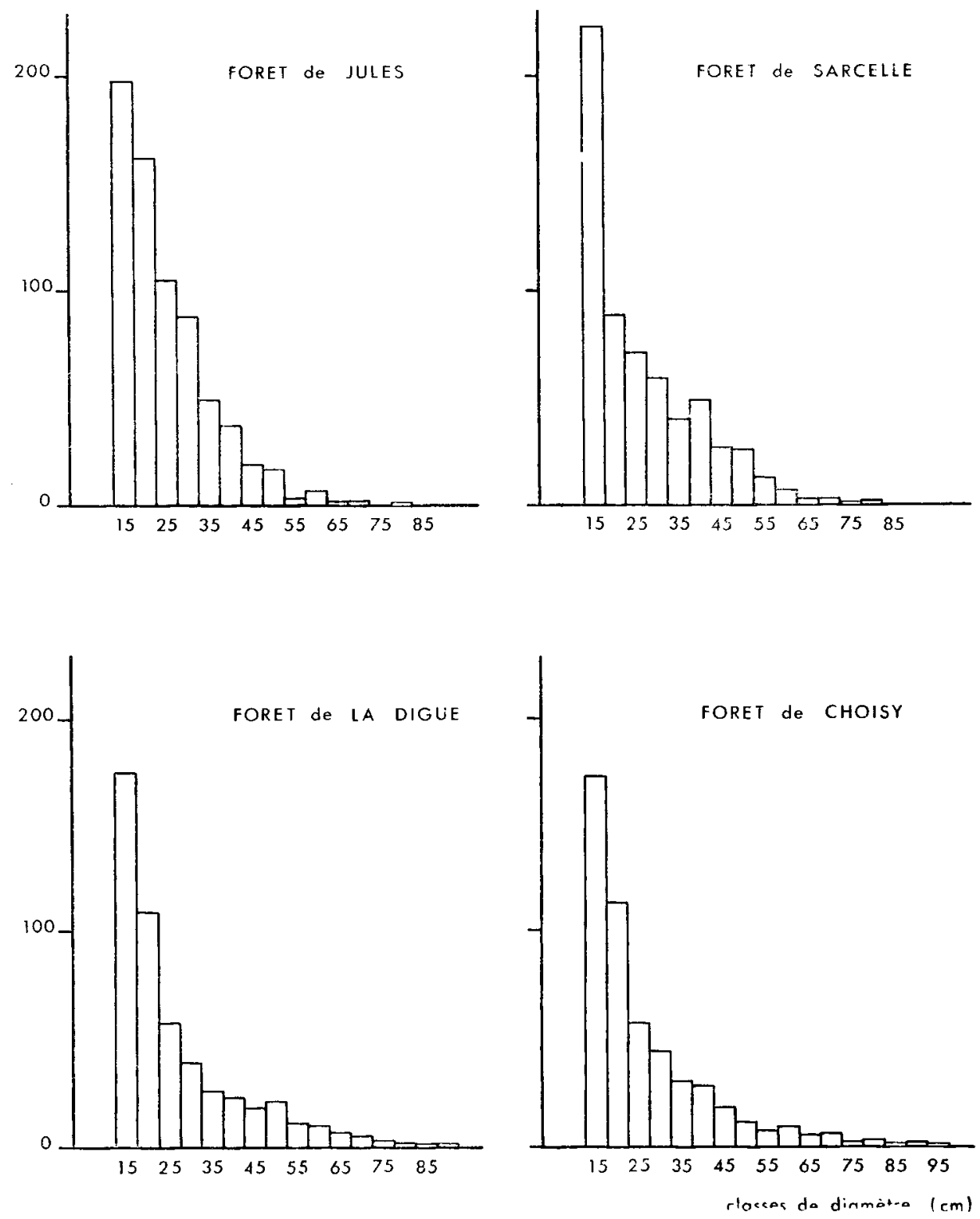

Fig. 2

Histogrammes de répartition du nombre de tiges en fonction des classes de diamètre pour chacun des 4 dispositifs expérimentaux.

Histogram of the tree number according to diameter size class for each of the four experimental sites. 
TABLeau 4

Date des premières opérations sylvicoles.

Date of the first silvicultural operations.

\begin{tabular}{|c|c|c|c|c|}
\hline Nature des travaux & Jules & Sarcelle & La Digue & Choisy \\
\hline $\begin{array}{l}\text { Nettoiement des par- } \\
\text { celles } \ldots \ldots \ldots \ldots \ldots\end{array}$ & Novembre 1979 & $\begin{array}{l}\text { Février- } \\
\text { mars } 1980\end{array}$ & Janvier 1981 & Novembre 1981 \\
\hline Martelage & Décembre 1979 & Avril 1980 & Février 1981 & Décembre 1981 \\
\hline $\begin{array}{l}\text { Exploitation } \ldots \ldots \ldots \\
\text { Dégagement des semis }\end{array}$ & $\begin{array}{c}\text { Janvier } 1980 \\
\text { Décembre } 1981 \\
\text { p. } 1 \text { et } 2 \\
\text { Février } 1982 \\
\text { p. } 3 \text { et } 4\end{array}$ & $\begin{array}{l}\text { Mai-juin } 1980 \\
\text { Septembre } 1981\end{array}$ & $\begin{array}{l}\text { Février } 1981 \\
\text { Août } 1982\end{array}$ & $\begin{array}{c}\text { Décembre } 1981 \\
\text { Juillet } 1983\end{array}$ \\
\hline
\end{tabular}

\subsection{Nature des observations}

Chaque parcelle de 2 ha a été subdivisée en 25 placettes carrées de $28 \mathrm{~m}$ de côté à l'aide d'un quadrillage interne dont le but était de faciliter les opérations d'inventaire et surtout de martelage. Les consignes de martelage étaient données dans chaque placette en fonction de l'inventaire initial et du type de coupe choisi. Ceci a permis dans une certaine mesure de régulariser le peuplement.

Les observations sur la régénération ont porté sur le comptage des semis et sur leur croissance.

Pour le comptage des semis, on a utilisé un taux d'échantillonnage de 1 p. 100 ce qui correspond à 100 placeaux circulaires de $2 \mathrm{~m}^{2}$ par parcelle de 2 hectares. Ces placeaux ont été placés à raison de 4 par placette à proximité des piquets délimitant le pourtour des placettes.

Pour les études de croissance on a choisi des semis, répartis dans des classes de hauteur variant de $10 \mathrm{~cm}$ à $100 \mathrm{~cm}$, à raison d'un plant par espèce à proximité de chaque placeau de comptage. On n'a pas pris de plants dans les placeaux situés à la périphérie de la parcelle ce qui donne un total de 64 plants par espèce et par parcelle.

Les placeaux de comptage des semis et de mesure de la croissance ont été mis en place progressivement d'avril 1981 à septembre 1982.

Le dispositif de Sarcelle a fait l'objet d'une étude microclimatique particulière. Des abris météorologiques simplifiés ont été installés dans chaque parcelle ainsi que sous le témoin et dans une clairière voisine. Ils permettent d'avoir des mesures hebdo- 
madaires des températures minimum et maximum ainsi que de l'évaporation mesurée aul «piche».

Ces mesures ne sont pas faites en permanence mais simplement pendant des périodes plus ou moins longues ( 6 mois à un an) à la suite des coupes. On a caractérisé le couvert restant après les coupes d'ensemencement, toujours dans le dispositif de Sarcelle, grâce à la perméabilité relative au rayonnement solaire calculée à partir de photographies hémisphériques (DuCrEY, 1975).

\section{Les coupes d'ensemencement}

Après quelques tâtonnements les consignes données à l'occasion des martelages étaient les suivantes : pour les coupes dites faibles, on enlève environ 10 p. $100 \mathrm{du}$ nombre de tiges choisies de préférence dans les divers et surtout dans l'étage dominé, pour les coupes dites fortes on enlève environ 30 p. $100 \mathrm{du}$ nombre de tiges toujours dans les divers mais à la fois dans l'étage dominé pour rehausser le couvert et dans l'étage dominant pour favoriser la pénétration de la lumière. On a cherché autant que faire se peut à régulariser au maximum le convert.

Cette exigence, d'ordre expérimental, est cependant fondamentale puisque le but de l'expérience est de rechercher des sylvicultures différentes favorisant ou bien les espèces d'ombre ou bien les espèces de lumière. Il est évident que cette décision n'est pas sans conséquences sur la manière dont on réalise les coupes. On recherche vraiment à homogénéiser le couvert pour donner des niveaux d'éclairement au sol proches de ce que l'on souhaite et aussi réguliers que possible sur l'ensemble d'une parcelle. Ceci nous a notamment conduits à ne pas enlever les plus gros divers dominants dès la première coupc.

Ceci n'empêchera pas dans la pratique si l'on recherche de manière raisonnce une sylviculture favorisant les espèces de lumière d'agir dès la coupe d'ensemencement très fortement dans les divers dominants à large houppier. Mais si on cherche impérativement à favoriser les espèces d'ombre il faudra assumer les conséquences d'un enlèvement lent et progressif du couvert.

\subsection{Conséquences sur la structure du peuplement}

La figure 3 donne les histogrammes de fréquence en fonction des classes de diamètre pour la moyenne des parcelles coupes fortes et coupes faibles. Pour les coupes fortes on a enlevé en moyenne 28 p. 100 des tiges à peu près dans toutes les classes de diamètre, sauf les très gros qui ont des cimes très développées et qui auraient provoqué de trop grosses trouées. Pour les coupes faibles, on a enlevé en moyenne 12 p. 100 du nombre de tiges surtout dans les classes 15 et 20 et un peu dans les classes 25 à 35 . Ceci correspond à des pourcentages de surface terrière enlevée de 20 p. 100 pour les coupes fortes et de 5 p. 100 dans les coupes faibles.

On observe d'assez grandes variations d'une parcelle à une autre ainsi que le montre le tableau 2. Ces variations sont dues d'une part à la structure initiale du peuplement : présence de trouées, prédominance de gros arbres ou au contraire d'arbres de petit diamètre, et d'autre part à une dérive dans le temps de la technique de martelage. 


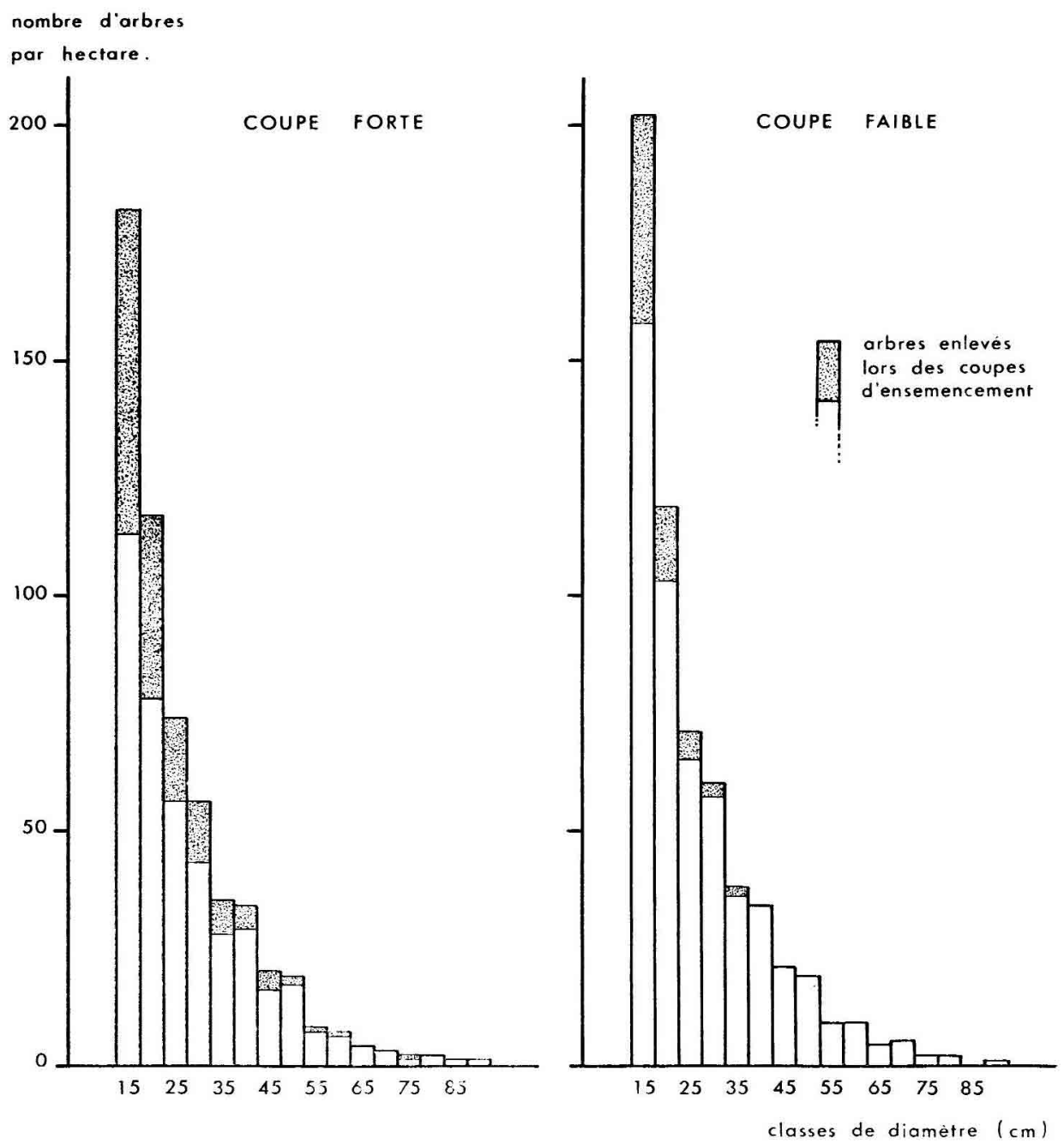

FIG. 3

Répartition, en fonction des classes de diamètre, du nombre de tiges enlevées au cours des coupes d'ensemencement fortes et faibles pour l'ensemble des 4 dispositifs. Histogram of diameter size class before and after felling according to the felling intensity : light and heavy (all sites confounded).

\subsection{Conséquences sur le microclimat forestier}

Le tableau 5 indique les résultats obtenus à Sarcelle pendant la période allant du 28 octobre 1981 au 19 mai 1982 et qui comprend une partie de saison sèche et une partie de saison humide. 
En ce qui concerne les températures, on observe les différences habituelles entre forêt et plein découvert, l'effet de tampon du couvert forestier étant très marqué, surtout au niveau de lamplitude journalière qui est deux fois plus forte en plein découvert que sous forêt. Les différentes parcelles éclaircies se situent bien entre ces deux extrêmes mais sont encore très proches de la forêt. Il en est de même pour l'évaporation hebdomadaire mesurée au piche. On n'a pas pu mettre en évidence de différences entre les deux types de coupe. L'absence de différences entre parcelles s'explique par le fait qu'il n'y a qu'un seul point de mesure (en principe le centre géométrique de la parcelle) qui ne correspond pas forcément aux conditions moyennes du couvert.

La perméabilité relative au rayonnement solaire (valeur pondérée tenant compte du type de temps et de différentes trajectoires du soleil au cours de l'année) est une valeur moyenne calculée sur 36 emplacements par parcelle. Les deux parcelles fortement éclaircies ont des perméabilités de 15,9 et $10,0 \mathrm{p}, 100$, tandis que celles faiblement éclaircies ont des valeurs égales à 7,1 p. 100 et 4,7 p. 100. Ces valeurs sont sans doute un peu sous-estimées par rapport à la réalité (biais dû à la technique), mais marquent bien les différences entre les coupes. On observe une bonne relation entre ces valeurs et la surface terrière des parcelles.

\section{Conclusion}

La forêt hygrophile de Guadeloupe est, par certains côtés, très originale. En effet, en raison de son caractère insulaire c'est une forêt qui est pauvre en espèces. Mais, inversement, certaines espèces, parmi celles qui nous intéressent ont une place prédominante dans cette forêt même si elles sont pratiquement inconnues ailleurs : le bois rouge carapate dont on a vu l'importance est endémique de la Guadeloupe et de la Dominique.

Par contre, certaines espèces comme l'acajou blanc ou le palétuvier jaune, sont présentes dans tout le bassin amazonien.

Toutes ces raisons font que la forêt de Guadeloupe présente une spécificité dans le monde tropical qui la rapproche des autres îles des Petites Antilles et à ce titre nos travaux ont valeur de modèle pour ces îles, mais qui ne la sépare pas entièrement non plus du continent américain.

Dans le prochain article nous nous intéresserons à l'installation ct à la croissance des semis pendant la période allant des coupes d'ensemencement aux premières coupes secondaires. Sur le terrain, les premiers résultats sont d'ores et déjà très spectaculaires et la vigueur des semis d'acajou blanc ainsi que l'étendue des taches de semis de bois rouge nous permettent d'être raisonnablement très optimistes quant à l'avenir de cette régénération naturelle.

Reçu le 2 mai 1984.

Accepté le 26 septembre 1984. 


\section{Summary \\ Investigations on controlled natural regeneration in the tropical rain forest of Guadeloupe}

\section{I. - Literatur review, forest conditions and elaboration of an experimental design}

The paper reviews literatur references on natural regeneration of tropical forests and presents basic informations on the tropical rain forest of Guadeloupe.

This forest has a particular structure among the tropical forest ecosystems, with a rather high frequency of certain timber species. For example, Dacryodes excelsa and Amanoa caribaea reach a mean frequency of 20.7 p. 100 and 12.6 p. 100 among the total number of trees. Other interesting timber species such as Richeria grandis, Simaruba amara, Symphonia globulifera, Byrsonima coriacea, Chimarrhis cymosa and Phoebe elongata occur less frequently.

All together, the cight mentionned species total 48 p. 100 of all trees and 64 p. 100 of the total basal area on an average.

Because of this particular composition, a control of natural regeneration for favouring the most valuable timber species seems to be promising. Methods close to the progressive felling, and to the tropical shelterwood system could be suited for this purpose.

An experiment was designed and carried out from 1979 through 1981. Based on an accurate description of the forest, the design involved 5 treatments including a control plot. Each plot unit had an area of 2 hectares except the control ( 1 ha).

The treatments were as follow :

1) heavy seed felling, secondary felling 3 years later and a final cut at 6 years;

2) heavy seed felling, secondary felling 4 years later and a final cut at 8 years;

3) light seed felling, two secondary fellings 3 and 6 years later and a final cut at 9 years;

4) light seed felling, two secondary fellings 4 and 8 years later and a final cut at 12 years.

The same design was replicated at 4 different sites.

It was intended to study the natural regeneration, the survival and the growth of seedlings according to the different species. The goal was also to use the variations in environmental conditions due to the different treatments, as a mean for revealing the ecological requirements of the eight tree species.

\section{Références bibliographiques}

AleXandre D.Y., 1977. Régénération naturelle d'un arbre caractéristique de la forêt de la Côte-d'Ivoire : Turraeanthus africana Pellegr. Oecologia Plantarum, 12 (3), 241-262.

Ashton P.S., 1978. The natural forest : Plant biology - Regeneration and tree growth, 180-215. In : Natural resources research XIV. Tropical forest ecosystems, U.N.E.S.C.O., Paris.

Aubreville A., 1929. Comment constituer une forêt tropicale de rapport. Revue de Botanique Appliquée, $560-568$.

Aubreville A., 1938. La forêt coloniale. Les forêts de l'Afrique occidentale française. Annales Acad. Sci. coloniales, tome 9, 244 p.

BARNARD R.C., 1950. Linear regeneration sampling. Malayan forester, 13 (3), 129-142. 
BEARD J.S., 1949. The natural vegetation of the Windward and Leew'ard islands. Clarendon Press, Oxford, $192 \mathrm{p}$.

Bell G.S., 1976. Notes on natural regeneration in Dominica, Windward Islands. Commonwealth forestry review, 55 (1), 27-36.

Bellouard, 1951. La régénération des forêts en A.O.F. Première conférence forestière interafricaine, Abidjan, 4-12 décembre 1951, 69-94.

Burgess P.F., 1968. An ecological study of the hill forests of the Malay Peninsula. Malayan Forester, 31 (4), 314-325.

Catinot R, 1965. Sylviculture en forêt dense africaine, $1^{\mathrm{re}}$ partic. Bois et forêts des tropiques, 100, 5-18.

CAtinol R., 1974. Le présent et l'avenir des forêts tropicales humides. Bois et forêts des tropiques. 154, 3-26.

Donis C., 1956. La forêt dense congolaise et l'état actuel de sa sylviculture. Bulletin agricole du Congo Belge, 47 (2), 261-294.

Douay J., 1954. Appréciation des possibilités de régénération d'une parcelle de forêt tropicale par comptage des préexistants. Bois et forêts des tropiques, 36, 11-19.

Ducrey M., 1975. Utilisation des photographies hémisphériques pour le calcul de la perméabilité des couverts forestiers au rayonnement solaire. 1. - Analyse théorique de l'interception. Ann. Sci. For., 32 (2), 73-92.

DuCrey M., 1975. Utilisation des photographies hémisphériques pour le calcul de la perméabilité des couverts forestiers au rayonnement solaire. II. - Etude expérimentale. Ann. Sci. For., 32 (4), 205-221.

Dulau, 1952. Le milieu physique et les aspects actuels de la végétation de la Guadeloupe. Thèse de phytosociologic non publiée. Dactylographiéc, 6 tomes, $480 \mathrm{p}$.

Duplaquet L., 1960. Le centre tropical de recherches forestières de Rio Piedras, à Porto-Rico, Rev. For. fr., 12 (7), 445-457.

Duss R.P., 1897. Flore phanérogamique des Antilles françaises. Annales de l'Institut colonial de Marseille. Imprimerie Prota frères, Mâcon, $656 \mathrm{p}$.

DE Foresta H., 1981. Aspects de la régénération forestière trois ans après coupe papetière. L'écosystème forestier guyanais. Etude et mise en valeur. Bulletin de liaison du groupe de travail C.T.F.T. - I.N.R.A. Muscum-O.R.S.T.O.M., n ${ }^{\circ}$ 3, avril 1981, 63-67.

Fournet J., 1978. Flore illustrée des phanérogames de Guadeloupe et Martinique. Publ. I.N.R.A., Paris, 1654 p.

Fox J.E.D., 1971. Natural regeneration of the kambui hills forest in castern Sierra Leone. Part I1. Methods of silvicultural treatment and analysis. Tropical Ecology, 12 (1), 1-23.

Fox J.E.D., 1972. Effets de l'utilisation accrue de la forêt naturelle sur le succès de la régénération naturelle à Sabah. Bois et forêts des tropiques, 141, 4-22.

Geollegue R.T., HuC R., 1979. Early stages of forest regeneration in Southeast Asia. Biotrop. Bogor. Indonesie, June 19-21 1979, 12 p.

GREBERT, 1931. Situation forestière de la Guadeloupe. Congrès de la production forestière coloniale et nord-africaine. Exposition coloniale internationale, Paris, 1931, G 128, $11 \mathrm{p}$.

LAmprecht H., 1976. De l'opportunité, des possibilités et des problèmes de la régénération naturelle en forêt tropicale. Journal forestier suisse, 127 (2), 91-97.

Lanly J.P., Clement J., 1979. Horizon 2000 - Superficie des forêts et des plantations sous les tropiques. Unasylva, 31 (123), 12-20.

Lasserre G., 1961. La Guadeloupe, Tome 1, La nature et les hommes. Thèse 1961. Edition KolodzIEJ, E.D.C.A., 1978, 448 p.

Lavauden L., 1934, 1935. La forêt équatoriale africaine. Son passé, son présent, son avenir. Actes et comptes rendus de l'Association Colonies-Sciences, 10 (114), 209-223; 11 (115), $1-8$.

Leroy DeVAl. J., 1976. Biologie et sylviculture de l'okoumé. Tome 1, La sylviculture de l'Okoumé, Publ. C.T.F.T., Paris, 355 p.

Little E.L., Woodbury R.O., Wadsworth F.H., 1974. Trees of Puerto Rico and the Virgin Islands. Second volume. Agriculture Handbook $n^{\circ}$ 449. U.S. Department of Agriculture. Forest service, $1024 \mathrm{p}$. 
Little E.L., Wadsworth F.H., 1964. Common trees of Puerto Rico and the Virgin Islands. Agriculture Handbook $\mathrm{n}^{\circ}$ 249. U.S. Department of Agriculture Forest service, $548 \mathrm{p}$.

LoNGwood F.R., 1962. Present and potential commercial timbers of the caribbean. Agriculture Handbook n" 207. U.S. Department of Agriculture. Forest service, 167 p.

Maudoux E., 1954. La régénération naturelle dans les forêts remaniées du Mayumbe. Bulletin agricole da Congo Belge, 45 (2), 403-421.

MaZUera H., 1979. Composition and growth of four to fifteen year old natural regeneration in the lower calima concession. Investigación forestal. Research report $n^{\circ} 46,2-27$. Publ. cellulosa y papel de Colombia S.A.

Nwoвoshi L.C., 1976. Problems and prospects of natural regeneration systems in the future management of the tropical moist forest for timber production. Document F.A.O., quatrième scssion, Comité de la mise en valeur des forêts dans les tropiques.

Ordeman R.A.A., 1972. L'architecture de la fort̂t guyanaise. Thèse O.R.S.T.O.M., Publ. C.N.R.S., AO 7787,247 p.

PérII P., 1969. Resultados preliminares de unos estudios sobre la regeneración natural espontanea en el bosque «el caimital». Revista forestal venezolana, 18, 9-21.

PRevost M.F., 1981. Recru de trois ans après coupe de type papetier. L'écosystème forestier guyanais. Etude et mise en valeur. Bulletin de liaison du groupe de travail C.T.F.T.1.N.R.A.-Muséum-O.R.S.T.O.M., n" 3, avril 1981, 68-81.

Richards P.W., 1952. The tropical rain forest. An ecological study. Cambridge. University Press, $450 \mathrm{p}$.

Rollet B., 1969. Etudes quantitatives d'une forct dense humide sempervirente de plaine de la Guyane vénézuélienne. Thèse doctorat, Faculté des Sciences, Toulouse, 473 p.

Rollet B., 1969. La régénération naturclle en forêt dense humide sempervirente de plaine de la Guyane vénézuélienne. Bois et forêts des tropiques, 124, 19-38.

Rollit B., 1983. La régénération naturelle dans les trouées : un processus général de la dynamique des forêts tropicales humides. Bois et forêts des tropiques, 201, 3-34; 202, 19-24.

Rostilar D.R., Lancastiz P.C., 1953. Historique et aspect actuel de la sylviculture en Nigeria. Bois et forêts des tropiques, 28, 3-12.

Schulz J.P., 1967. La regeneración natural de la selva mesofitica tropical de Surinam después de su aprovechamiento. Instituto forestal latino-americano de investigación. MeridaVenezuela. Boletin $\mathrm{n}^{\circ} 23,3-27$.

Stehle H., 1936. Flore de la Guadeloupe et dépendances. Tome 1. Essai d'écologie et de géographie botanique, $284 \mathrm{p}$.

Strane H., 1937. Flore de la Guadeloupe et dépendances. Tome 2. Catalogue des phanérogames et fougères, $236 \mathrm{p}$.

Strule H., 1946. Les types forcstiers des îles caraïbes. Deuxième partie. The caribbean forester, vol. 7, suppl. 337-709.

Stevinson N.S., 1928. Aménagement des forêts d'Acajou dans le Honduras britannique. Revue de Botanique appliquée, 281-283.

Synnort T.J., Kemp R.H., 1976. Choisir la meilleure méthode de régénération. Unasylva, 28 (112-113), 73-89.

TAYlor C.J., 1954. La régénération de la forêt tropicale dense dans l'oucst africain. Bois et forêts des tropiques, 37, 19-26.

Webb L.J. et al., 1972. Regeneration and pattern in the subtropical rain forest. J. Ecol., 60, 675-695.

Winfmore T.C., 1978. Gaps in the forest canopy, 591-615. In : Tropical trees as living systems. Tomlinson and Zimmermann (eds), New York, Cambridge University Press.

Wilten W., 1955. Aspects de la sylviculture au Mayumbe. Bulletin agricole du Congo Belge, 46 (2), 319-328.

Wyлtт-Smith J., 1963. Manual of Malayan Silviculture for Inland Forests. Vol. 1. Malayan Forest Records, 23, Forest Department, Malaya. 